were of remarkable size, quite colossal in comparison with shallow water or littoral forms. Of the three species of Colossendeis, two of which are described as new, the smallest has a span of $14 \mathrm{~cm}$. between the tips of its outstretched legs, while the largest has an extent four times as great. A new genus (Scæorhynchus) has been established for a species with a span of I9 $\mathrm{cm}$., a gigantic size as compared with the dimensions of its nearest allies. The most abundant species of Nymphon is the largest of that extensive genus, and one species of a new genus (Pallenopsis) is more than twice as large as any of the species of allied genera, such as Pallene, which are known only from the littoral zone. It is further interesting to note that in a number of forms the visual organs (ocelli) are either rudimentary and destitute of pigment, or are entirely absent. In Pallenopsis, however, the ocelli are relatively of unusually great size. The species of Scæorhynchus and Colossendeis show clearly from anatomical evidence the complete independence of the accessory legs and the first pair of ambulatory legs, as had been already proved by Dohrn from embryological data. In all cases the palpi and accessory legs are supplied with nerves from the same ganglion, and this latter shows in the adult no indication of being composed of two coalesced ganglia. But Dohrn states that there are in the larvæ of Achelia two ganglia. This question is of great interest, having a direct bearing on the affinities of these Pycnogons with the Arachnids. Mr. Wilson describes ten species, of which one half are given as new, and with figures.

New Zealand Desmids, -As a contribution to our knowledge of the pretty green unicellular algæ known as Desmids, which are to be met with in New Zealand, Mr. Maskell's paper in the recently-published rolume of the Transactions of the New Zealand Institute is most welcome. It would seem to render more than probable the idea that these minute algæ are to a large extent cosmopolitan. The author is evidently under great disadvantage as to identifying the species he meets with, but this is to a great extent done away with by his fairly careful descriptions and accurate (as to outline) figures. $\mathrm{He}$ enumerates between sixty and sixty-five species, some of which are very noteworthy and fine additions to the list of Desmids; thus Aptogonum undulatum is a highly interesting new species. Triploceras bidentatum is not only a very distinct, but also a very noble, new species, and equally distinct as a species is his Closterium selencum. Doubtless a more prolonged search in fresh localities will enable the author to add many old and new species to the list. He may feel sure that his further researches will be looked for with interest by those working at the freshwater algæ in Europe.

Protoplasm Stained whilst Living.-Mr. L. F. Henneguy publishes the result of some experiments made on living infusoria, in which he confirms the observations of Brandt, made in 1879 , that an aqueous solution of aniline brown, known in commerce as Bismarck brown, will give an intense brownish. yellow colour to the protoplasm of the infusoria without in any way interfering with their enjoyment of life. The coloration first appears in the vacuoles of the protoplasm, then this latter is itself stained, the nucleus being most generally not at first coloured, and so being made more conspicuous. Experiments made on vegetable protoplasm seemed to exhibit the same result.

\section{LARGE TELESCOPES 1}

THE small amount of work accomplished with large telescopes has often been the subject of unfavourable comment. Thi criticism applies with especial force in America, where there are nearly a dozen telescopes having an aperture of a foot or over, besides two of the largest size now in course of construc tion, and two of twenty-six and twenty-four inches aperture which are unmounted and have been for several years perfectly useless. Among so many it seems as if one might be spared for a trial of the following plan, which, if successful, would produce at a small expense far more work than could be obtain $d$ with a mounting of the usual form.

Suppose that the telescope is placed horizontally at right angles to the meridian, and that a plane reflector inclined to its axis by $45^{\circ}$ is placed in front of it. This reflector may revolve around an axis coimciding with that of the telescope. Such a mounting has been used in transit instruments, and gives mauch I By Edward C. Pickering, communicated by the author. satisfaction in the meridian photometer of the Harvard College Observatory. The principal difficulty with a large instrument would lie in the flexure of the reflector. This difficulty has how. ever been overcome in a great measure in reflecting telescopes by various ingenious devices. In the present case, since the refiector rotates only around one axis instead of two, the problem is much simplified. A slight motion at right angles of perhaps $5^{\circ}$ would be a great convenience, as will be shown below, and would probably be insufficient to materially affect the flexure. It may be said that it is more difficult to make a plane surface than one that is curved. But the principal effect of a slight curvature would be to change the focus of the telescope, the aberration being much less than the effect of the varying flexure. Let us admit, however, that the best definition cannot be obtained, in considering the purposes to which such an instrument could be applied without disadvantage.

Many advantages will be apparent on comparing such a mounting with an equatorial. Great steadiness would be secured, since the mirror would be the only portion moved, and this would be placed directly upon a low pier. Instead of a large and expensive dome which is moved with difficulty, the mirror would be protected by a small shed, of which the roof could be easily removed. It would therefore be opened and ready for use in a very short time, and would quickly take the temperature of the surrounding air. The object-glass would be mounted directly upon a second pier, and, as it would not be moved, would be in very little danger of accident. The tube could be made of tin or other inexpensixe material, as its flexure is of no importance. It could easily be protected from the changes of the tem. perature so troublesome in the tube of a large equatorial. If preferred it might even be exhansted of air, or filled with hydro. gen, and the effect of the changes of temperature thus greatly reduced.

The eyepiece could be mounted on a third pier, and would be so far distant horizontally from the mirror and ohject-glass that there is no reason that it should not be inclosed in a room which may be warmed. The comfort in winter of working in a warm room will be appreciated by those who have used a large telescope in a cold climate. The result is sure to be an increased precision in the observations, and a possibility of prolonging them over longer intervals. A similar effect is produced by the constant direction of the line of sight. No especial observing chair is needed. There is no limit to the size of the attachments which may be made to the eyepiece, since they need not be moved. This is a great advantage in certain spectroscopic and photometric measurements. A strong wind interferes seriously with many observations, as it is impossible to make a telescope so stiff that it will not be shaken by sudden gusts. In the plan here proposed the mirror alone is exposed, and its surface is too small to give trouble.

By means of a long handle the position of the mirror may be regulated from the eyerend, and the declination of the object observed read by small telescopes. If the mirror can be moved at right angles to the meridian $5^{\circ}$ from its central position, an object at the equator may be followed for forty minutes, and other objects for a longer period. Without this motion an object may be followed for three or four minutes by moving the eyepiece alone. Clockwork may be applied to the mirror, or less easily to the eyepiece. The focal length may be increased almost indefinitely if desired, and certain advantages will be thus attained in the diminution in the defects of the object-glass, although those of the reflector will not be affected. If the telescope is to be erected at a great elevation the advantages of the present plan are at once apparent. Many nights of observation would be secured which otherwise would be lost owing to the wind and cold. The simplicity in the construction of the build. ing would be a great advantage, as a large dome in so exposed a situation would be kept free from snow with much difficulty, and might be a source of danger in winter storms. If found impracticable to observe during the winter, it would be possible to have a duplicate mounting below, and remove the lens and mirror from one to the other.

It is evident that the saving of cost would be very great, not only in the observatory building and dome, but in the tube, observing chair, clockwork, \&c.

If a reflector could be constructed whose surface was the portion of a paraboloid whose abscissa equalled that of the focus, the instrument eovld be much simplified. No object-glass would then be required, the reflector taking the place both of mirror and lens. All the light intercepted by the objective would thus 
be saved, and but a single surface need be adjusted and corrected. With the advance in mechanical methods this does not seem wholly impracticable, especially with a mirror of long focus. Since the final correction must always be made by hand, it makes but little difference what is the exact form of the surface.

In any case it would be a great advantage that the mirror could be reground, repolished, or resilvered without moving it from its place. It would only be necessary to place it horizontally, and the grinding machinery could be kept permanently near it. If plane, the perfection of its form could also be tested at any time by setting it on edge, and viewing the image it reflected by a collimating eyepiece attached to the large telescope. Another method would be to place a heliotrope a few hundred yards to the north or south of it, and the light from this would form an excellent artificial star, available whenever the sun shone.

The greatest advantage is the rapidity with which observations could be made. No more time would be lost in identification than with a transit instrument, so that a large number of objects could be examined in the course of a single hour. Any one who has worked with a large telescope knows how much time is lost in opening and closing the dome and in finding and identifying minute objects.

Let us now consider to what purposes a large telescope mounted as suggested might be applied.

I. Sweeping. For the discovery of new objects this mounting presents especial advantages. It might be used for the detection of new double stars, of nebulæ, of red stars, or of objects having singular spectra, as planetary nebulæ, banded stars, and variables of long period. Suppose that the field of view had a diameter of somewhat over one minute of time, and that a small motor was attached to the mirror which would move it uniformly over $5^{\circ}$ in declination in this time, and then bring it quickly back to its first position. The observer would then have presented to him a series of zones $5^{\circ}$ long and one minute wide. The sweeps should overlap by a small amount, so that the entire region could be covered in a single evening. The observer could have a few seconds rest between each zone, while the motion of the mirror was reversed. If an object of interest was suspected, it could be located by merely noting the time at which it was seen. The right ascension would be given directly, and the declination would be found by interpolation from the time of beginning and ending the sweep. An examisation of the object and a determination of its exact location should be made on another evening.

2. Measures of position. For many purposes positions could be determined with this instrument as in a transit circle. It would generally be better however to make the measures differential, leaving the mirror at rest and observing the transits of the object to be determined and of two or more companion stars. The method of the ring micrometer might be employed, or some modification of that with inclined lines. In the latter case the zero of position could be found by the transit of preceding stars, by setting the reticule by a divided position circle, or perhaps better by keeping it in a fixed position, determining the direction of the lines once for all, and applying a correction for the declination of the object observed. Stars could be compared differing nearly a degcee in declination, as the eyepiece could be moved without danger of disturbing the reticule. For the same reason the star could be followed for three or four minutes, and its transit over a great number of wires observed. It is here assumed that the distortion produced by the mirror is not very great. A slight distortion would do little harm, as it would be the same for all stars of equal brightness. If the stars differ greatly in brightness, the observer should determine his personal equation between them in any case, and the same operation would eliminate the effect of the distortion. The large aperture of the instrumeat would permit the observation of stars quite beyond the reach of any meridian circle. The faintest asteroids could thus be readily measured, and could probably be followed in many cases on successive evenings to their stationary points. Zones of stars could be observed very conveniently for the formation of charts or catalogues, for the discovery of asteroids, stars with large proper motion, \&c.

Probably the definition could not be sufficiently good for the measurement of the closer double stars, but if clockwork was attached, faint companions could be measured, or approximate positions of the coarser pairs determined very rapidly. The positions of nebulæ could also be observed with a view to detecting their proper motion. Stars having a large proper motion might be observed, and the observations so arranged that any very large parallax would be detected. A similar search for a large parallax of variable star,, short-period binaries, minute planetary nebulæ, or stars having singular spectra, might lead to interesting results. The argument that no ordinary star is very near does not apply to such objects.

3. Spectroscopy. The increased dimensions which could be given to the spectroscope, and its steadiness, would compensate in a great measure for a defect in definition. By Zöllner's reversion spectroscope the slit might be dispensed with, and also the necessity of clockwork. So many stars could be observed in a single evening that systematic errors could be in a great measure eliminated, and as the spectroscope would not be moved, we should have a great assurance that the deviations were real. Of the 6000 nebulæ hitherto discovered we know nothing of the spectrum of more than 300 or 400 , while the observation of all the others with a large horizontal telescope would not be a very formidable undertaking. It would also be interesting to observe the spectra of all the clusters. It is possible that some may consist of stars having singular spectra, or even of disconnected nebulous masses, in fact forming clusters of planetary nebulæ. The interesting discovery by Dr. Copeland that Burnham's double nebula in Cygnus is gaseous, shows the same tendency to aggregation in these bodies as in stars. Observations of the spectra of all the red stars and variables would also prolsably lead to interesting results.

4. Photometry. Should the instrument be devoted to photometry numerous problems suggest themselves. Variable stars could be observed near their minimum when too faint to be identified with an equatorial without great loss of time. Faint stars in zones or faint companions to bright stars could be measured very rapidly. The relative light of all the asteroids would be an interesting problem. Many coarse clusters appear to consist of stars of nearly equal brightness. Their light compared with their distances apart might aid our study of their formation. Another useful investigation would be to measure the brightness of all the nebulæ.

In the application of physics to astronomy doubtless many other problems will suggest themselves. Thus nu satisfactory results have been obtained in the attempt to measure the heat of the stars with the tasimeter. The use of this instrument would be vastly simplified if it was placed on a solid pier near the ground, was not moved during the observation, and could be perfectly protected from other changes of temperature than those which it was intended to measure.

As either of the problems proposed above would occupy the time of a telescope for at least one year, it is obvious that there could be no difficulty in keeping such an instrument occupied indefinitely.

The horizontal monnting is especially adapted to an elevated position, and would permit the use of a telescope where an equatorial mounting would be quite impracticable. On the other hand, to an amateur, or for purposes of instruction, an instrument which could be set quickly from one object to another, and where the observers need not be exposed to the cold, would offer many advantages. The impossibility of observing far from the meridian would be less important with a large instrument, where the number of objects to select from is very great.

There are certain purposes to which this mounting could not be advantageously applied. The study of close double stars and other objects requiring long examination and very perfect definition could be better left to other instruments. The sun, moon, and planets can also generally be better observed off the meridian. If, however, the entire time of an instrument can be employed to advantage, and it can collect several times as much material as an instrument of the usual form, it is no evidence against its trial that there are certain problems to which it cannot be advantageously applied.

The working force required for such an instrument should consist of at least one observer, an assistant to record, and a number of copyists and computers to prepare the working lists, reduce the observations, prepare them for the press, and read and check the proof-sheets. A large volume of valuable observations could thus be produced every year, which would require at least double the time and money to produce by the same telescope mounted equatorially. 'The difference in the amount of work will be evident when we compare the number of obj scts observed with a transit instrument per night, with those observed with an equatorial. A hundred objects in various declinations might be examined in a single evening, while it is seldom that the same number could be identified and measured by an equatorial in a week. 